\title{
Correction to: Constructing High-Order Dynamic Functional Connectivity Networks from Resting-State fMRI for Brain Dementia Identification
}

Chunxiang Feng, Biao Jie, Xintao Ding, Daoqiang Zhang, and Mingxia Liu

\section{Correction to: \\ Chapter "Constructing High-Order Dynamic Functional Connectivity Networks from Resting-State fMRI for Brain Dementia Identification" in: M. Liu et al. (Eds.): Machine Learning in Medical Imaging, LNCS 12436, https://doi.org/10.1007/978-3-030-59861-7_31}

In a former version of this paper, the CERNET Innovation Project (NGII20190621) was missing from the Acknowledgement section. This has been corrected. 